\title{
PERCEPÇÃO DE BLOQUEIO/FACILITAÇÃO DE COMUNICAÇÃO: UM EXPERIMENTO
}

\author{
Lucianne Dalla Bemardina * \\ Maria Márcia Bachion ** \\ Emília Campos de Carvalho** \\ Luís de Souza ***
}

BERNARDINA, L. D.; BACHION, M. M.; CARVALHO, E. C. de; SOUZA, L. de. Percepção de bloqueio/facilitação de comunicação: um experimento. Rev.latino-am.enfermagem, Ribeirão Preto, v. 4, $\mathrm{n}^{\circ}$ especial, p. 61- 70, abril 1996.

Considerando-se o referencial de FORREST (1983). realizamos um experimento com o objetivo de verificar como os entrevistados (alunos do $6^{\circ}$ semestre de graduação em enfermagem) percebem um diálogo levando-se em conta 12 itens de avaliação (clima de abertura, interesse, mudança de assunto, questões direcionadas, desaprovação do assunto, tranqüilidade, tensão, aconselhamento, reconhecimento de sentimentos. reflexão de assuntos, aprovação e fuga de assuntos). O tratamento constou de 02 entrevistas com diferentes proporções de categorias facilitadoras $(F)$ e bloqueadas $(B)$ da comunicação: Grupo A - 55\% de categoria $F$ e 45\% de categoria B: Grupo B $75 \%$ de categoria $B$ e $25 \%$ de categoria $F$. Como resultados encontramos que não houve diferença estatisticamente significante para os grupos, em nenhum dos itens de avaliação propostos. Ressaltamos o fato da entrevistadora ser conhecida dos entrevistados, a temática ser de fácil abordagem e o nível de relação ser do tipo horizontal.

UNITERMOS: comunicação, comunicação bloqueadora, comunicação facilitadora

\footnotetext{
* Enfermeira do Hospital das Clínicas da Faculdade de Medicina de Ribeirão Preto da Universidade de São Paulo - Ex. bolsista de Iniciação Científica, CNPq/USP.

** Docentes da Escola de Enfermagem de Ribeirão Preto da Universidade de São Paulo

*** Docente da Faculdade de Medicina de Ribeirão Preto da Universidade de São Paulo
} 


\section{INTRODUÇÃO}

O estudo do comportamento verbal é viabilizado pelo emprego de modelos teóricos, oriundos da comunicação social. Dentre eles destacamos o modelo de FORREST (1983), proposto como estratégia para o indivíduo explorar a si e a seus problemas. Esse modelo envolve nove categorias que facilitam a comunicação e outras nove que bloqueiam a mesma.

As categorias facilitadoras têm várias funções: estimular a verbalização do entrevistado: promover um clima de abertura: fornecer elementos que permitam uma compreensão da situação: transmitir interesse e empatia.

As categorias bloqueadoras permitem desde apenas o direcionamento do diálogo, fuga do assunto até a centralização dos valores do entrevistador, depreciação dos sentimentos do entrevistado, ou mesmo a desaprovação formal, como um reforço negativo para o comportamento comunicativo. Tal modelo foi desenvolvido tendo-se em vista o relacionamento entre enfermeiro e paciente, contudo, acreditamos que ele possa ser aplicado considerando outras díades.

No âmbito da enfermagem BACHION (1991); CAVICHIOLLO \& CARVALHO (1992); CARVALHO et al (1992) empregaram tal modelo com pacientes portadores de doenças crônico-degenerativas.

As categorias facilitadoras são descritas por FORREST (1983) como:

01. Declarações amplas ou questões de abertura: permitem ao entrevistado direcionar a conversação, determinando seu foco.

02. Encorajamento para o entrevistado continuar: indica interesse e compreensão do que o mesmo está dizendo.

03. Refletindo: todas ou partes das declarações do entrevistado são repetidas ou ligeiramente reformuladas para encorajar a continuação.

04. Dividindo as observações, verbalizando as percepções com o entrevistado: podem focalizar o estado físico ou emocional, do entrevistado; este é convidado a verificar, corrigir ou elaborar sobre as observações do entrevistador. Diz respeito à verbalização do entrevistador em decorrência da percepção de um comportamento do entrevistado, sem emitir uma interpretação.

05. Reconhecendo os sentimentos do entrevistado e como ele se sente: encoraja o entrevistado a continuar expressando sentimentos, sem demonstrar julgamento sobre eles. A verbalização do entrevistador é relacionada à verbalização do entrevistado sobre seus sentimentos, independente se o entrevistador pensa do mesmo modo.

06. Reconhecendo a presença do entrevistado: estão incluídos nesta subcategoria cumprimentos, o nome do entrevistado ou substantivos que o designarem.

07. Dando informação: incluem as respostas às perguntas, diminuindo ansiedades, 
ou a apresentação de fatos que o entrevistado quer ou precisa saber. Estabelece a verdade.

08. Clarificando, deixando mais claro o significado ou requerendo do entrevistado que clarifique o significado: ambigüidades ou mal-entendidos e motiva o entrevistado a continuar.

09. Verbalizando pensamentos subentendidos ou sentimentos: expressa o que o entrevistado está insinuando ou sugerindo, em lugar do que tem sido dito. Ajuda o entrevistado a tomar-se mais ciente dos seus pensamentos e sentimentos e auxilia o entrevistador a verificar impressões.

\section{As Categorias bloqueadoras englobam, segundo o autor, os seguintes tipos:}

01. Tranqüilizando com clichê ou comentários estereotipados: são comentários comuns, dados automaticamente, que tendem a mostrar ao entrevistado o desinteresse do entrevistador ou falta de compreensão ou própria ansiedade.

02. Aconselhamento: comentários do entrevistador, passando por cima da decisão tomada pelo entrevistado, por imposição das próprias opiniões e soluções, ao invés de auxiliar o mesmo a explorar, chegando às conclusões.

03. Aprovando ou concordando: comentários ou opiniões que focalizam os valores e sentimentos do entrevistador, impostos sobre a livre expressão do entrevistado.

04. Pedindo explicação: perguntas feitas ao entrevistado para avaliar e explicar sentimentos ou ações: com freqüência envolve "por que" em questões que podem intimidar o entrevistado.

05. Desaprovando ou discordando: o entrevistador emite julgamento negativo às ações, pensamentos ou sentimentos do entrevistado, introduzindo valores que podem intimidar o entrevistado promovendo conformidade para aprovação do entrevistador.

06. Despreciando: indicam que as experiências do entrevistado não são únicas ou importantes, transferindo para um foco distante do mesmo.

07. Defendendo: comentários de proteção ou desculpas em vez de permitir que o entrevistado expresse suas próprias opiniões e sentimentos.

08. Mudando de assunto: introdução um novo tópico não relacionado, tomando a liderança da conversação de entrevistado, que não pode fazer qualquer tentativa para que suas necessidades sejam conhecidas.

09. Fechando o questionamento: focaliza questões fechadas que podem limitar as respostas do entrevistado e sugerir a procura de entrevistado por uma resposta específica.

Considerando os dois grupos citados, ressalta BACHION (1991) que não se deve imprimir às subcategorias facilitadoras o conceito de adequacidade e às bloqueadoras o conceito de inadequacidade, face à complexidade do processo de interação humana. 
No presente estudo, buscaremos alcançar o objetivo de verificar como os entrevistados (alunos do $3^{\circ}$ ano de graduação em enfermagem) percebem um diálogo, estimulado com duas diferentes proporções de categorias facilitadoras e bloqueadoras da comunicação, cuja temática versa sobre a realização do procedimento de infusão endovenosa de soros, em laboratório e em área hospitalar.

\section{MÉTODO}

\section{- População e Amostra}

Os dados foram coletados entre alunos de enfermagem que estavam cursando 0 $6^{\circ}$ semestre da Escola de Enfermagem de Ribeirão Preto da Universidade de São Paulo e manifestaram concordância em participar da investigação.

\section{- Procedimentos: Coleta de Dados}

Uma das pesquisadoras se identificava ao aluno e, a seguir, solicitava a concordância do mesmo para participar de uma entrevista gravada, com o objetivo de avaliar o desenvolvimento de uma interação, cujo tema abordava aspectos da aprendizagem prática em enfermagem. Ao final do desenvolvimento da entrevista gravada ( $1^{\mathrm{a}}$ etapa) era solicitado ao aluno que efetuasse a avaliação da mesma ( $2^{\mathrm{a}}$ etapa).

\section{$1^{\text {a }}$ etapa}

Seguindo-se uma seqüência aleatória (sorteada previamente), cada aluno foi identificado em pertencente ao Grupo A ou Grupo B. Os alunos de cada grupo foram entrevistados de acordo com um roteiro composto por uma seqüência pré estabelecida de comportamentos verbais, com diferentes proporções de categorias facilitadoras e bloqueadoras:

Para o grupo A a seqüência foi composta de 55\% Categorias Facilitadoras e $45 \%$ Categorias Bloqueadoras da Comunicação. O roteiro seguido, com as respectivas categorias, esta apresentado abaixo:

\section{Roteiro para Grupo A}

01. Como foi a sua primeira técnica de soroterapia? ( $F_{1}$ - questão de abertura).

02. (Repetir a última frase em forma de pergunta) $\left(F_{3}\right.$ - reflexão).

03. Hum... continue... ( $F_{2}$ - encorajamento).

04. Essa técnica não tem muita importância, existem outras mais complexas... ( $\mathrm{B}_{6}$ depreciando).

05. Você vai participar do Congresso Brasileiro de Enfermagem? ( $B_{8}$ - mudança de assunto). 
06. E quando você foi para o hospital em estágio e executou a técnica de soroterapia, não vai me dizer que ficou ansiosa(o) e tremeu toda(o)? ( $\mathrm{B}_{5}$ - desaprovando).

07. (Mencionar o nome do entrevistado) ( $F_{6}$-reconhecendo a presença). Observei que ficou (verbalizar o comportamento), ao relatar sua experiência... ( $F_{4}$ - dividindo observações).

08. Você geralmente procura revisar a técnica quando sente que não está muito apta(o) para realizá-la? ( $B_{9}$ - questão fechada).

Para o Grupo B a seqüência foi composta por 75\% Categorias Bloqueadoras e 25\% Categorias Facilitadoras da Comunicação. Segue-se o roteiro utilizado.

\section{Roteiro para o Grupo B}

01. Você aprendeu a técnica de soroterapia? ( $B_{9}$ - questão fechada).

02. Você instalou muito soro em campo de estágio? ( $\mathrm{B}_{9}$ - questão fechada).

03. Como foi a sua primeira técnica de soroterapia? ( $F_{1}$ - questão aberta).

04. É... continue... ( $F_{2}$ - encorajamento).

05. Essa técnica não tem muita importância, sondagem vesical é mais importante... ( $\mathrm{B}_{6}$ - depreciando).

06. Mas no final, tudo dá certo, é só diluir os medicamentos e pronto $\left(B_{1}\right.$ tranqüilizando com clichê).

07. Você participou da Semana de Enfermagem? ( $\mathrm{B}_{8}$-mudança de assunto).

08. Você geralmente executa as técnicas em laboratório antes de ir para os estágios? (B $_{9}$-questão fechada).

09. Acho que um treinamento antes de ir para o hospital é bom para se aprimorar... $\left(B_{2}\right.$ - aconselhamento).

\section{$2^{\mathrm{a}}$ etapa}

Após a entrevista era solicitado ao aluno que avaliasse a sensação que teve durante a entrevista, através da escala abaixo:

\begin{tabular}{|l|l|l|l|l|l|}
\hline ITENS DE AVALIAÇÃo & PÉSSIMO & RUIM & NEUTRO & BOM & ÓTIMO \\
\hline 01. Clima de Abertura & & & & & \\
\hline 02. Interesse & & & & & \\
\hline 03. Mudança de Assunto & & & & & \\
\hline 04. Questões Direcionadas & & & & & \\
\hline 05. Desaprovação do Assunto & & & & & \\
\hline 06. Tranqüilidade & & & & & \\
\hline 07. Tensão & & & & & \\
\hline 08. Aconselhamento & & & & & \\
\hline 09. Reconhecimento de sentimentos & & & & & \\
\hline 10. Reflexão de assuntos & & & & & \\
\hline 11. Aprovação & & & & & \\
\hline 12. Fuga de Assuntos & & & & & \\
\hline
\end{tabular}


Foram atribuídos "scores" de 1 a 5 a cada alternativa de resposta para os itens adotados quanto a percepção do desenvolvimento das entrevistas, representadas da seguinte forma:

\begin{tabular}{|l|c|c|c|c|c|}
\hline SCORES & 01 & 02 & 03 & 04 & 05 \\
\hline RESPOSTAS & PÉSSIMO & RUIM & NEUTRO & BOM & ÓTIMO \\
\hline
\end{tabular}

Para efetuarmos a análise dos dados utilizamos o teste não-paramétrico de WILCOXON-MANN-WHITNEY (WOOLSON, 1987) para comparar amostras independentes.

\section{RESULTADOS}

Foram sujeitos do presente estudo, 24 alunos matriculados no $6^{\circ}$ semestre do curso de graduação em enfermagem (47,05\%).

Os dados obtidos mediante a aplicação do instrumento para a avaliação das sensações dos entrevistados no decorrer da entrevista estão dispostos em dois quadros distintos. O QUADRO I contém as respostas do grupo A (submetido a aproximadamente $55 \%$ de emissões facilitadoras e $45 \%$ de bloqueadoras).

QUADRO I - PERCEPÇÃO DO GRUPO A SOBRE A ENTREVISTA ITENS DE AVALIAÇÃO PÉSSIMO RUIM NEUTRO BOM ÓTIMO

\begin{tabular}{|l|c|c|c|c|c|}
\hline ITENS DE AVALIAÇÃO & PÉSSIMO & RUIM & NEUTRO & BOM & ÓTIMO \\
\hline 01. Clima de Abertura & - & 01 & 02 & 07 & 02 \\
\hline 02. Interesse & - & - & 02 & 05 & 05 \\
\hline 03. Mudança de Assunto & - & 01 & 05 & 04 & 02 \\
\hline 04. Questões Direcionadas & - & - & 04 & 05 & 03 \\
\hline 05. Desaprovação do Assunto & - & - & 11 & - & 01 \\
\hline 06. Tranqüilidade & - & 01 & 02 & 05 & 04 \\
\hline 07.Tensão & - & 02 & 07 & 03 & - \\
\hline 08. Aconselhamento & - & - & 06 & 04 & 02 \\
\hline 09. Reconhecimento de sentimentos & - & - & 03 & 05 & 04 \\
\hline 10. Reflexão de assuntos & - & 01 & 03 & 06 & 02 \\
\hline 11. Aprovação & - & - & 05 & 05 & 02 \\
\hline 12. Fuga de Assuntos & 01 & 01 & 08 & 02 & - \\
\hline TOTAL & $\mathbf{0 1}$ & $\mathbf{0 7}$ & $\mathbf{5 8}$ & $\mathbf{5 1}$ & $\mathbf{2 7}$ \\
\hline
\end{tabular}


Notamos no Quadro I a prevalência das percepções nos campos neutro (58) e bom (51), sendo neutro para os itens: desaprovação do assunto (11); fuga de assuntos (08); tensão (07); aconselhamento (06); mudança de assunto (05); questões direcionadas (04); reconhecimento de sentimento e reflexão de assuntos (03); clima de abertura e interesse (02). Para o campo bom obtivemos os itens: clima de abertura (07); reflexão de assuntos (06); interesse, questões direcionadas, tranqüilidade, reconhecimento de sentimentos e aprovação (05); mudança de assunto e aconselhamento (04); e fuga de assuntos (02). Dado o quase "equilíbrio" de emissões facilitadoras e bloqueadoras dirigidas a este grupo, com leve predomínio das primeiras, podemos dizer que estes achados estão dentro do esperado, de acordo com o referencial teórico.

O QUADRO II contempla os resultados obtidos no grupo B (submetidos a aproximadamente $75 \%$ de emissões bloqueadoras e $25 \%$ de facilitadoras).

QUADRO II - PERCEPÇÃO DA ENTREVISTA PELO GRUPO B

\begin{tabular}{|l|c|c|c|c|c|}
\hline ITENS DE AVALIAÇÃO & PÉSSIMO & RUIM & NEUTRO & BOM & ÓTIMO \\
\hline 01. Clima de Abertura & - & 01 & 02 & 08 & 01 \\
\hline 02. Interesse & 02 & - & 04 & 03 & 03 \\
\hline 03. Mudança de Assunto & 02 & 02 & 06 & - & 02 \\
\hline 04. Questões Direcionadas & 01 & 01 & 03 & 05 & 02 \\
\hline 05. Desaprovação do Assunto & 02 & - & 06 & 03 & 01 \\
\hline 06. Tranqüilidade & 01 & - & - & 04 & 07 \\
\hline 07.Tensão & - & 01 & 08 & 02 & 01 \\
\hline 08. Aconselhamento & 01 & - & 07 & 02 & 02 \\
\hline 09. Reconhecimento de sentimentos & - & 01 & 07 & 03 & 01 \\
\hline 10. Reflexão de assuntos & 02 & 01 & 03 & 04 & 02 \\
\hline 11. Aprovação & 01 & 01 & 04 & 03 & 03 \\
\hline 12. Fuga de Assuntos & 02 & 02 & 07 & 01 & - \\
\hline TOTAL & $\mathbf{1 4}$ & $\mathbf{1 0}$ & $\mathbf{5 7}$ & $\mathbf{3 8}$ & $\mathbf{2 5}$ \\
\hline
\end{tabular}

A percepção do grupo B predominou nos campos neutro (57) e bom (38); notamos aqui um aumento das percepções dos campos péssimo e ruim em relação ao grupo anterior. Obtivemos para o campo neutro os itens: tensão (08); aconselhamento, reconhecimento de sentimentos e fUga de assuntos (07); mudança de assunto e desaprovação do assunto (06); interesse e aprovação (04); questões direcionadas e reflexão de assunto (03); clima de abertura (02); o item tranqüilidade teve freqüência zero (0). Para o campo bom evidenciaram-se os itens: clima de abertura (08); questões direcionadas (05); tranqüilidade e reflexão de assuntos (04); interesse, desaprovação do assunto, reconhecimento de sentimentos e aprovação (03); tensão e aconselhamento (02); fUga de assuntos (01): o item mudança de assunto não foi mencionado. Segundo as proposições de FORREST (1986), as emissões bloqueadoras trariam ao entrevistado sentimentos de clima 
fechado, desinteresse, direcionamento, julgamento negativo do entrevistador, desaprovação por parte do entrevistador entre outros sentimentos negativos. Isto aconteceu para alguns entrevistados, que assinalaram os campos péssimo e ruim para alguns itens.

Lembramos que junto às verbalizações dos entrevistados ocorreram os comportamentos não-verbais (gestos, expressões, tom de voz, etc.) que podem ter suavizado as emissões bloqueadoras. Além disso, como o entrevistador era colega da faculdade dos entrevistados, pode não ter sido obtido o grau de formalidade ou verticalidade da relação que usualmente ocorre entre profissional-paciente. Assim, estes fatores devem ser levados em conta ao pensarmos no impacto das categorias bloqueadoras numa situação de diálogo desta natureza, o que não existia em nenhum dos estudos onde o modelo de FORREST (1986) foi empregado.

Ao analisarmos estatisticamente se a percepção de cada item de avaliação foi diferente para os grupos $A$ e $B$, obtivemos os seguintes resultados dispostos no QUADRO III.

QUADRO III - VALOR DE p PARA CADA ITEM DE AVALIAÇÃO DA

\begin{tabular}{|l|c|}
\hline ITEM DE AVALIAÇÃo & VALOR $\mathbf{p}$ \\
\hline 01. Clima de Abertura & 0,790 \\
\hline 02. Interesse & 0,123 \\
\hline 03. Mudança de Assunto & 0,093 \\
\hline 04. Questões Direcionadas & 0,464 \\
\hline 05. Desaprovação do Assunto & 0,719 \\
\hline 06. Tranqüilidade & 0,223 \\
\hline 07. Tensão & 0,713 \\
\hline 08. Aconselhamento & 0,225 \\
\hline 09. Reconhecimento de sentimentos & 0,034 \\
\hline 10. Reflexão de assuntos & 0,413 \\
\hline 11. Aprovação & 0,715 \\
\hline 12. Fuga de Assuntos & 0,334 \\
\hline
\end{tabular}

Como o teste englobava 12 itens, O nível de significância passou a ser 0,05/12 tendo-se, então, o nível final igual a 0,004, de acordo com as diretivas de Bonferroni (SNEDECOR \& COCHRAN, 1980). Assim, não foi encontrada diferença estatisticamente significante na percepção dos entrevistados dos grupos A e B, para nenhum dos itens de avaliação.

Tal fato corrobora nossa crença de que outros fatores, ainda não levados em conta em estudos anteriormente realizados, interfiram na percepção que os entrevistados tiveram acerca dos itens de avaliação. Além disso, a temática era de fácil abordagem, não implicando em questões de valor moral ou de experiência intimista que trouxessem repercussões profundas para aqueles que vivenciaram tais situações. 


\title{
CONSIDERAÇÕES FINAIS
}

A interação interpessoal é um fenômeno complexo, tendo sido aqui abordado apenas através de aspectos facetários, Ao serem desenvolvidos, os modelos de comunicação, incluindo os que tratam do contexto interpessoal, representam uma realidade circunstancial e específica, No caso do modelo de FORREST (1986), foi desenvolvido para uma situação de relação "terapeuta-paciente", visando a ajuda, $\mathrm{Na}$ presente pesquisa, dadas as diferenças de circunstâncias e sujeitos, as subcategorias bloqueadoras não reforçaram o esperado pela literatura.

As proporções de emissões facilitadoras e bloqueadoras adotadas presentemente causaram as mesmas percepções para os grupos A e B.

É importante ressaltar que o entrevistador era conhecido dos entrevistados e a temática de fácil abordagem.

$\mathrm{Na}$ interação os elementos estavam em posição de igualdade, Assim, acreditamos que para haver bloqueio da exploração do entrevistado, na situação, seria necessário adotar subcategorias bloqueadoras mais intimidatórias tais como $\mathrm{B}_{4}$ (pedindo explicação), $\mathrm{B}_{5}$ (desaprovando), juntamente com subcategorias facilitadoras de menor abertura, como $F_{2}$ (encorajamento), $F_{6}$ (questão de reconhecimento) e $F_{7}$ (dando informações).

Parece-nos oportuno sugerir novos estudos, abordando interações em outros tipos de díades, bem como, outros arranjos (combinações) de comportamentos facilitadores e bloqueadores.

\section{PERCEPTION OF THE BLOCKING/FACILITATING COMMUNICATION: AN EXPERIMENT}

\begin{abstract}
Based on the reference work of FORREST (1983), we conducted an experiment in order to determine how interviewees (6th semester nursing undergraduate course) perceive a dialogue, taking into consideration 12 items of evaluation (opening, interest, changing subject, directed questions, disapproval of the subject, tranquility, tension, counseling, recognizing feelings, reflection about the topics, approval, and flight from the topics). Treatment consisted of interviews with different proportions of categories of facilitating $(F)$ and blocking $(B)$ communication: Group A - 55\% F category and 45\% B category; Group B - 75\% B category and 25\% F category, There was no statistical difference between groups in terms of any of the proposed evaluation items. We emphasize the fact that the interviewer was known by interviewees. The topic was easy to approach and the level of relationship was of the horizontal type.
\end{abstract}

UNITERMS: communication, categories of blocking communication, categories of facilitating communication 


\section{PERCEPCIÓN DE BLOQUEO/FACILITACIÓN DE LA COMUNICACIÓN: UN EXPERIMENTO}

Considerando el referencial de FORREST (1983), realizamos un experimento con el objetivo de verificar como los entrevistados (alumnos del VI semestre de pregrado en enfermería) perciben un diálogo teniendo en cuenta 12 ítems de evaluación (momento emocional del inicio, interés, cambios del tema, preguntas direccionadas, tensión, aconsejamiento, reconocimiento de sentimiento, reflexión de asuntos, negación del tema, tranquilidad, aprobación y escape del tema). El manejo contó de 02 entrevistas con diferentes niveles de categorías, facilitadoras $(F)$ y bloqueadoras $(B)$ de la comunicación: Grupo A - 55\% de la categoría $F$ y $45 \%$ de la categoría B, Grupo $B-75 \%$ de la categoría $B$ y $25 \%$ de la categoría $F$. Como resultados encontramos que no hubo diferencia estadísticamente significativa para los grupos en ninguno de los ítems de evaluación propuestos. Resaltamos el hecho de que la entrevistadora es reconocida por los entrevistados, la temática es de fácil abordaje y el nivel de relación es de tipo horizontal.

UNITERMOS: comunicación, comunicación bloqueadora, comunicación facilitadora

\section{REFERÊNCIAS BIBLIOGRÁFICAS}

01. BACHION, M. M. Análise do padrão de comunicação oral entre equipe de enfermagem e parturientes. Ribeirão Preto, 1991. p. 147. Dissertação (Mestrado) - Escola de Enfermagem de Ribeirão Preto, Universidade de São Paulo.

02. CARVALHO, E. C.; BACHION, M. M.; BRAGA, M. R. Comunicação oral com pacientes hematológicos: comportamentos facilitadores e bloqueadores. In: CONGRESSO BRASILEIRO DE ENFERMAGEM. 45. Brasília, outubro de 1992, p. 128. Programa, Brasília, 1992 (mimeografado).

03. CAVICHIOLLO, S. A.; CARVALHO, E. C. Análise da interação entre equipe de enfermagem e pacientes com distúrbios de comunicação. In: SIMPÓSIO BRASILEIRO DE COMUNICAÇÃO EM ENFERMAGEM, Ribeirão Preto, maio de 1992. Anais. Ribeirão Preto, 1992. p. 114-28.

04. FORREST, D. Analysis of nurses' verbal communication with patient. Nurs.Paper: perspectives in nursing, v. 15, n. 3, p. 48-57, 1983.

05. SNEDECOR, G. W.; CO, W. G. Statistical methods. 7. ed. Ames: lowa State University, 1980. p. 507.

06. WOOLSON, R. F. Statistical methods for the analysis of biomedical data. New York: John Wiley, 1987. p. 513. 